\title{
VARIAÇÕES GRAFEMÁTICAS EM TRÊS TRATADOS MEDIEVAIS PORTUGUESES
}

Rita de Cássia Ribeiro de Queiroz*

RESUMO: Os três tratados medievais - "Dos benefícios de Deus", "Livro da consciência e do conhecimento próprio" e "Da amizade e das qualidades do amigo", compõem a tradição portuguesa do texto ascético-místico "Castelo Perigoso", compilado em dois manuscritos alcobacenses: ALC 199 e ALC 214, pertencentes à Biblioteca Nacional de Lisboa. A partir do estudo das variações grafemáticas que as duas versões apresentam, analisar-se-á alguns aspectos que marcam a ortografia portuguesa medieval.

PALAVRAS-CHAVE: Variação Grafemática, Português Medieval, Literatura Religiosa.

RÉSUMÉ: Les trois traités médiévaux - "Dos benefícios de Deus", "Livro da consciência e do conhecimento próprio" e "Da amizade e das qualidades do amigo", font partie de la tradition portugaise de l'oeuvre ascétique-mystique "Castelo Perigoso", qui est compilée dans les deux manuscrits alcobacenses: ALC 199 et ALC 214, appartenant à la Bibliothéque Nationale de Lisbonne. À partir d'étude de les variations graphémátiques qui présent les deux versions, quelques aspects de l'orthographie médiéval portugaise seront analisés.

MOTS-CLÉ: Variation Graphémátique, Portugais Médiéval, Littérature Religieuse.

*Professora Adjunta do Departamento de Letras e Artes - UEFS 


\section{CONSIDERAÇÕES INICIAIS}

A obra "Castelo Perigoso", tradução de uma longa epístola francesa, escrita por Frère Robert, monge cartuxo, para sua prima, Soeur Rose, freira da Ordem Fontevrault, encontra-se entre as obras do período medieval português.

Em Portugal foi traduzida no Mosteiro de Alcobaça, existindo atualmente dois códices de posse da Biblioteca Nacional de Lisboa. ${ }^{1}$ A versão portuguesa constitui, na realidade, uma adaptação livre do original francês, pois não há uma correspondência precisa que possa ter originado a tradução.

A obra "Castelo Perigoso" contém sete tratados, assim designados: 1 Castelo Perigoso; 2 Dos benefícios de Deus; 3 Livro da consciência e do conhecimento próprio; $4 \mathrm{Da}$ amizade e das qualidades do amigo; 5 Das penas do inferno; 6 Das alegrias do paraíso; 7 Livro dos três caminhos e dos sete sinais do amor embebedado.

A partir da edição dos segundo, terceiro e quarto tratados: "Dos benefícios de Deus", "Livro da consciência e do conhecimento próprio" e "Da amizade e das qualidades do amigo" (QUEIROZ, 2002), apresentar-se-á as variações grafemáticas que figuram intra e entre os dois manuscritos alcobacenses: ALC 199 e ALC 214.

Diante desta perspectiva, realizou-se três edições - uma semidiplomática, uma crítica e uma modernizada dos três tratados (QUEIROZ, 2002). Para o estudo das variações grafemáticas, foi eleita a edição semidiplomática, cuja interferência do editor ocorreu somente quanto ao desdobramento das abreviaturas, sendo a mais conservadora e a que permite que se detecte elementos que possibilitam uma análise da scripta do texto.

No entanto, é importante esclarecer que este tipo de estudo, ou seja, da scripta do texto, só é possível a partir de edições críticas de caráter conservador. Ao se eleger este tipo de edição, levou-se em consideração o que diz Tavani (1988, p. 35): 
(...) a constatação de que cada texto é um produto histórico, no qual se refletem a situação pessoal do autor, a sua concepção de mundo, seus conflitos sócio-econômicos, suas experiências existenciais, seus conhecimentos teóricos e práticos, o grau de sua adesão a todas as convenções do seu tempo e a coletividade a que pertence.

Seguindo esta orientação, buscou-se oferecer o texto mais próximo possível do original, tentando com isso chegar ao português corrente no período medievo português.

\section{O CONTEÚDO DOS TRÊS TRATADOS}

O segundo tratado, Dos Benefícios de Deus, assim como o primeiro, Castelo Perigoso, foi traduzido do francês. Contudo, as Horas da Crũ, em verso francês, foram suprimidas da tradução portuguesa. Esse tratado desdobra, amplamente, um trecho do primeiro acerca da comunhão (capítulo 47). O próprio autor, a partir de uma nota no capítulo 69 acentua o fato, levando-se a supor ser ele o autor do primeiro tratado. Nos capítulos 70 a 81 são apresentados os doze frutos espirituais do Santíssimo Sacramento, voltados para a Paixão.

O terceiro tratado, Livro da Consciência e do Conhecimento Próprio, traz conselhos do autoconhecimento. São abordados os seguintes temas: alma enamorada de Deus (capítulo 83), vaidade do mundo (capítulos 84 e 85) e conselhos espirituais (capítulos 86 a 88). A partir do capítulo 89 passa-se a tratar do autoconhecimento. No capítulo 91, há uma passagem referente aos sofrimentos de Jesus. Esse trecho remete ao primeiro tratado, no qual, o autor, em sete capítulos (40 a 46), refere-se à Paixão de Cristo.

O quarto tratado, Da Amizade e das Qualidades do Amigo, é o mais breve de todos. Apresenta-se em oito capítulos, sendo cinco dedicados às qualidades que se deseja num amigo: discrição, 
bondade, boa consciência, modéstia, fidelidade e que "nom seja sanhudo nem bravo". Sem isso, a amizade não será preservada.

\section{A GRAMÁTICA DO PORTUGUÊS MEDIEVAL}

A variação da escrita do texto medieval é constante, pois até então não havia uma normatização ortográfica, prevalecendo a representação da letra tanto no sentido de "sinal gráfico" quanto no sentido de "pronúncia", ou seja, "som".

Pêro de Magalhães de Gândavo (1981, p.9-10) diz:

As letras que se costumão muitas vezes trocar hu)as por outras, e em que se cometem mais vicios nesta nossa linguagem, são estas que se seguem, conuem a saber, $c, s, z$, e isto nace de não saberem muitos a differença que ha de hu)as às outras na pronunciação.

Muitos estudiosos do português medieval afirmam que, durante esse período, os livros eram copiados, freqüentemente, pelo processo do ditado para vários copistas ao mesmo tempo, os quais cometiam erros e diversificavam a grafia ao sabor do ouvido e da ignorância. Assim, mesclavam formas mais arcaicas com as da sua época em decorrência da falta de informações. Esse período da língua portuguesa é considerado fonético e, segundo Williams (1986, p. 33):

Cabe ressaltar que, oriundos de uma cultura escrita em Língua Latina, cabia aos copistas e/ou escribas representar muitos novos sons que não existiam em latim, sendo obrigados, portanto, a adaptar velhas grafias ou a inventar novas.

Michaëlis de Vasconcelos (1946, p. 33) afirma que eles realmente escreviam o que falavam, às vezes de forma perfeita ou imperfeita, de acordo com os vinte e cinco caracteres do alfabeto herdado. 


\subsection{AS VARIAÇÕES GRAFEMÁTICAS}

Serão analisadas as variações vocálicas e as variações

\begin{tabular}{|c|c|}
\hline Manuscito 199 & Mamscito 214 \\
\hline 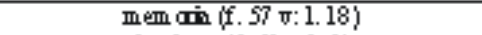 & memoren (f. 44 r: 1.12) \\
\hline hgrim as if. $98 \mathrm{r}: 1.1$ ) & lhgremas (f. $44 \mathrm{r}: 1$ 16) \\
\hline Gisto (f. $.8 \mathrm{~N}: 1.1)$ & deto (f. $44 \pi: 1.16$ ) \\
\hline cellistrinl if. $58 \pi: 1.18$ ) & ilistrinll (f.45r: 19) \\
\hline seirtemo if $.59 \mathrm{\pi}: 1.9$ ) / se temo (f. $59 \mathrm{\pi}: 1.10$ ) & ssetimo (f. $45 \pi: 1$ 19) / ssetemo if. $45 \pi: 1$. \\
\hline mellux if. 99 \%: 1.10 ) & mithar (f. $45 \pi: 1.20$ ) \\
\hline alegize (f. 60 \%: 1.19 ) & agrise if. $46 \pi: 1.25$ ) \\
\hline perturaill $(f .62 \pi: 117)$ & perdmanell (f. $48 \mathrm{r}: 1.26$ ) \\
\hline tribulhçónes (f. $63 \mathrm{r}: 1$ 10, 1.15 ) & trebuhf oै कै (f. $48 \pi: 113,1.17$ ) \\
\hline fexerom (f. $63 \pi: 1.8$ ) & frxeram (f. 49 r: 1.5$)$ \\
\hline degiplin (f. $65 \mathrm{r}: 1.16)$ & decelin if $.50 \mathrm{r}: 120$ ) \\
\hline
\end{tabular}

\begin{tabular}{|c|c|}
\hline Manuscrito 199 & Manscito 214 \\
\hline 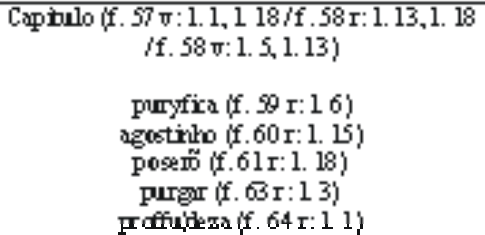 & 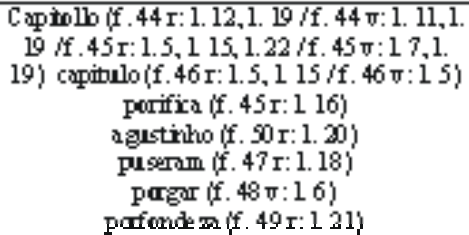 \\
\hline
\end{tabular}

As vogais mediais simples alternam-se na passagem do latim para o português com uma boa freqüência. Essa oscilação poderia ser uma variação gráfica livre; poderia ser uma representação da variante dialetal fônica de um mesmo vocábulo; ou seria uma indecisão de como grafar o segmento fônico. 


\begin{tabular}{|c|c|}
\hline Manucrito 199 & Mamusciito 214 \\
\hline pe (f. $59 \pi: 121)$ & pa (f. $46 \mathrm{r}: 17)$ \\
\hline fodarento if $.600: 111$ ) & fedorerto (f. $46 \pi: 118)$ \\
\hline 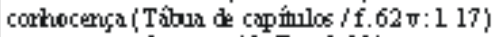 & contecercis $(f .49 \mathrm{r}: 1.19)$ \\
\hline conteçença (f. $\overline{3} \pi: 1.22)$ & \\
\hline contoçimeto if . $63 \mathrm{\pi}: 1.16$ ) conthe simento if. & contecimerto (f. $48 \pi: 12$ ) conthecimeto (f. \\
\hline $62 \pi: 120$ ) corthe in do (f. $63 \pi: 1.18$ ) & $49 \mathrm{r}: 113,1.16)$ \\
\hline propósito (f. 64 \%: 1.20 if. $65 \mathrm{r}: 1.1$ ) & sssyto if. $50 \mathrm{r}: 15$ \\
\hline
\end{tabular}

\begin{tabular}{|c|c|}
\hline Manusciito 199 & Mamuscrito 214 \\
\hline 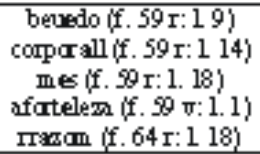 & $\begin{array}{l}\text { bebado (f. } 45 \mathrm{r}: 1.21 \text { ) } \\
\text { comporell (f. } 45 \pi: 1.2 \text { ) } \\
\text { mas if. } 45 \pi: 1.5 \text { ) } \\
\text { afortallem (f. } 45 \pi: 1.10 \text { ) } \\
\text { remin if. } 49 \pi: 1.10 \text { ) }\end{array}$ \\
\hline
\end{tabular}

\section{Variações entre os grafemas $<\mathrm{i}>/<\mathrm{j}>/<\mathrm{y}>$}

\begin{tabular}{|c|c|}
\hline Manuscino 199 & Manusciito 214 \\
\hline oytruso (Tíbun de c apínulos /f. 59 \%: 1.20 ) & oytrouo (f. $46 \mathrm{r}: 1.5,1.6)$ \\
\hline otmo (f. $59 \pi: 120)$ & \\
\hline justrimentos (Tíbun de capíllos) & \\
\hline 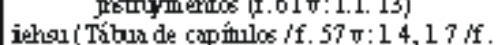 & johonu if $44 \mathrm{r} \cdot 12$ if $44 \pi \cdot 1221$ \\
\hline 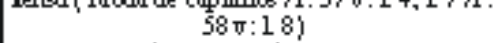 & 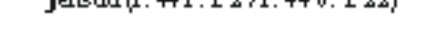 \\
\hline juferro (Tábun de cophulos if. $63 \mathrm{r}: 1.7$ ) & inferno (f. $48 \pi: 1.11$ ) \\
\hline & tyradas (f. $44 \mathrm{r}: 1.7$ ) \\
\hline latim (f. $57 \pi: 113$ ) & Intym (f. $44 \mathrm{r}: 1.8$ ) \\
\hline Aqui (f. $57 \pi: 1.15)$ & Aqy (f. $44 \mathrm{r}: 1.9$ ) \\
\hline $\begin{array}{l}\text { pindoso (f. } 58 \mathrm{r}: 1.1) \\
\text { matioso (f. } 58 \mathrm{r}: 1.7 \text { ) }\end{array}$ & $\begin{array}{l}\text { Pradoso (f. } 44 \mathrm{r}: 1 \text { 15) } \\
\text { Mayosso (f. } 44 \pi: 1 \text { i) }\end{array}$ \\
\hline amigo (f. $58 \mathrm{r}: 19$ ) & amyguo (f. $44 \pi: 1.3)$ \\
\hline enterirame if if. $58 \mathrm{r}: 1.9$ ) & enteyram ete (f. 44 \%: 1.3 ) \\
\hline $\begin{array}{c}\text { exce krticimo (f. } 58 \mathrm{r}: 1.3 \text { ) } \\
\text { ginh (f. } 58 \text { r: } 1.13 \text { /f. } 58 \text { \%:1. } 10 \text { ) }\end{array}$ & $\begin{array}{l}\text { excelertiosem o (f. } 44 \text { r: } 1.19 \text { ) } \\
\text { sinda (f. } 44 \text { \%: } 1.6 \text { /f. } 45 \text { r: } 1.3 \text { ) }\end{array}$ \\
\hline frito if. $58 \mathrm{r}: 1.18 / \mathrm{f} .58 \mathrm{\pi}: 1$ 10) fruyto if. 58 & fryto if. $44 \pi: 1.11,112,1.19,120 / f$. \\
\hline r: $119 /$ f. $58 \pi: 1.14$ ) & 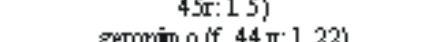 \\
\hline $\begin{array}{l}\text { gerorym o (f. } 58 \pi: 17) \\
\text { minto if } 58 \pi: 1.8)\end{array}$ & $\begin{array}{l}\text { gerorim o (f. } 44 \pi: 1.22) \\
\text { trrito if } 45 \mathrm{~s}: 1.1)\end{array}$ \\
\hline samtarbrogio (f. $58 \pi: 1$ 12) & sartanlrogro (f. $45 \mathrm{r}: 1.3$ ) \\
\hline & \\
\hline
\end{tabular}


Nestes exemplos, o fonema / i / aparece representado poligraficamente por $\langle\mathrm{i}\rangle,\langle\mathrm{j}\rangle$ e $\langle\mathrm{y}\rangle$.

2.1.1.2 Vogais nasais

\begin{tabular}{|c|c|}
\hline Manus ctio log & Manuscrite $11 d$ \\
\hline 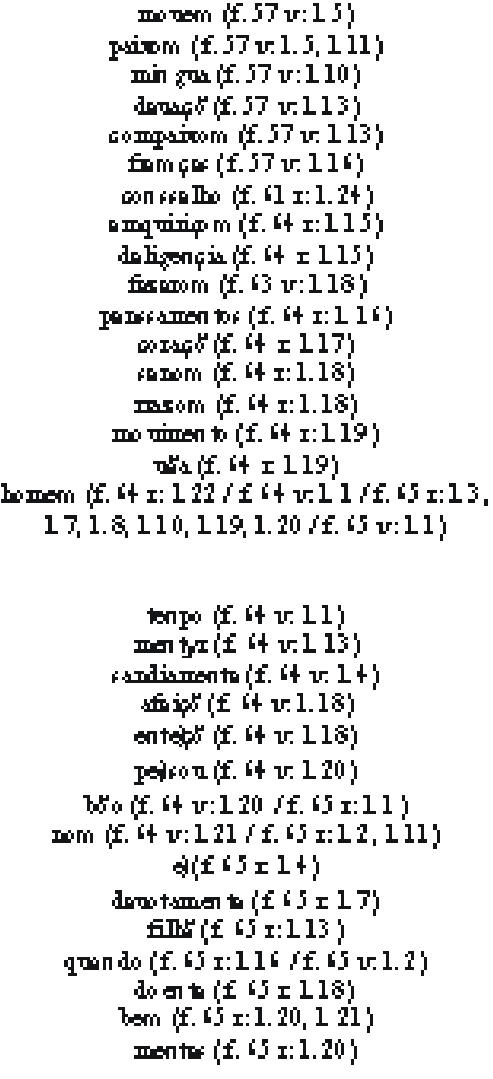 & 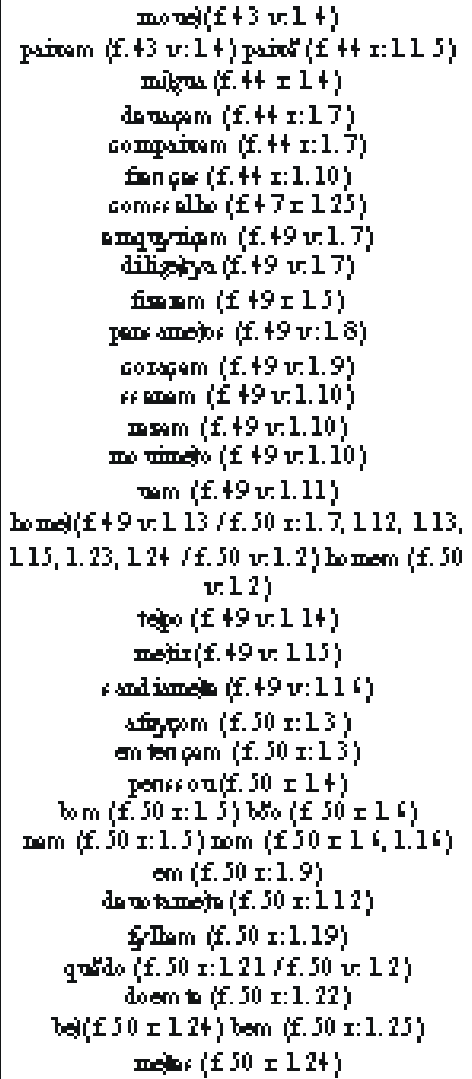 \\
\hline
\end{tabular}


Os exemplos acima mostram que a scripta das vogais nasais apresentam uma variação grafemática, cuja nasalidade é marcada

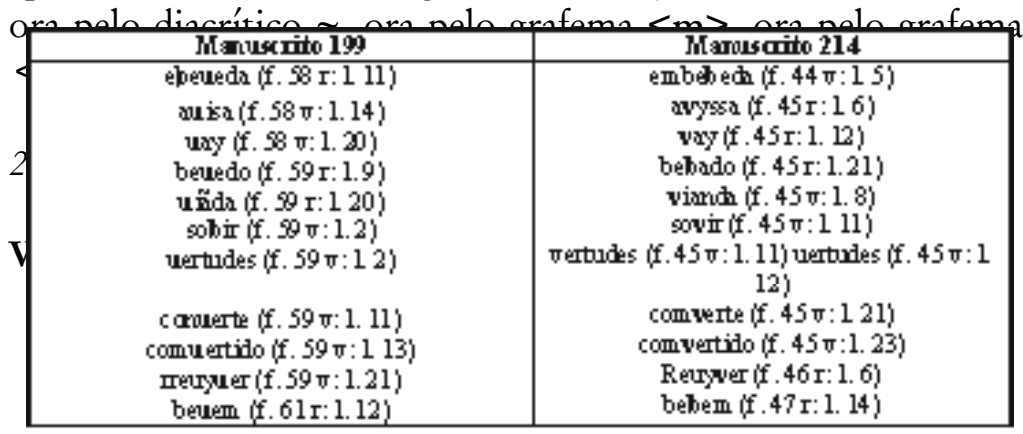

As consoantes: oclusiva bilabial sonora / b / e a fricativa labial sonora / v / são representadas, graficamente, pelos grafemas $\langle\mathrm{b}\rangle,\langle\mathrm{u}\rangle$ e $\langle\mathrm{v}\rangle$. Esta variação aponta, segundo Leão (1983, p. 54), para uma confusão existente entre / b / e /B / nos dialetos setentrionais portugueses. São variações que documentam a cadeia

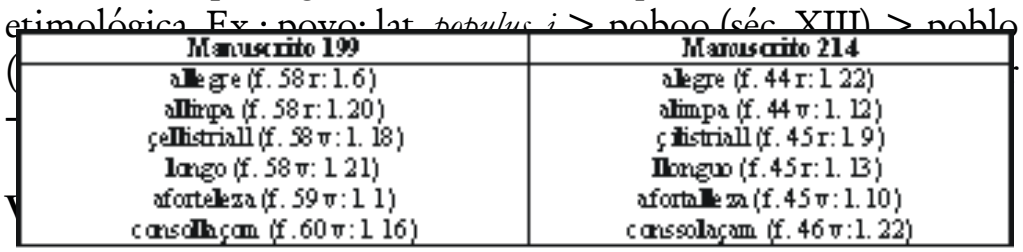




\begin{tabular}{|c|c|}
\hline Manucrito 199 & Mamusciito 214 \\
\hline $\begin{array}{l}\text { for if. } 99 \pi: 1.20 \text { ) } \\
\text { fiflo (f. } 61 \pi: 1.9 \text { ) } \\
\text { ffime if. } 65 \mathrm{r}: 1.15 \text { ) }\end{array}$ & $\begin{array}{l}\text { ffor (f. } 46 \mathrm{r}: 1.6 \text { ) } \\
\text { filho if } .47 \pi: 17 \text { ) } \\
\text { fire if. } 50 \mathrm{r}: 1.20 \text { ) }\end{array}$ \\
\hline
\end{tabular}

Variações entre os grafemas $<\mathrm{f}>$ e $<\mathrm{ff}>$

A simplificação do grafema $<11>$ para $<1>$ e do grafema $<\mathrm{ff}>$ para $<\mathrm{f}>$ decorre de um processo etimológico: na

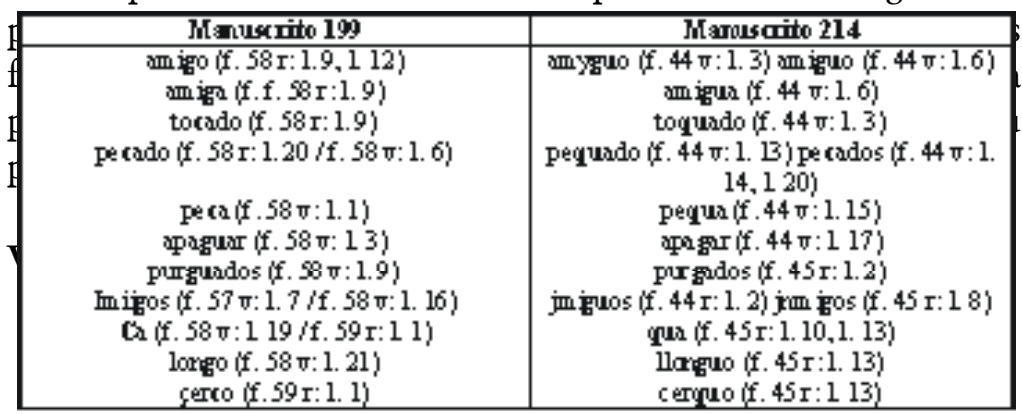

Tanto o grafema $\langle\mathrm{g}\rangle$ seguido das vogais $\langle\mathrm{a}\rangle,\langle\mathrm{o}\rangle$ e $\langle\mathrm{u}\rangle$, quanto o grafema $<$ gu $>$ seguido por $\langle$ e $>$ e $<\mathrm{i}\rangle$ representam a oclusiva velar sonora, assim como os grafemas $\langle\mathrm{c}\rangle$ diante de $\langle\mathrm{a}\rangle,\langle\mathrm{o}\rangle$ e $\langle\mathrm{u}\rangle$ e $\langle\mathrm{qu}>$ diante de $\langle$ e $>$ 
e $<\mathrm{i}>$ representam a velar surda. Os exemplos acima mostram que o grafema $<\mathrm{u}>$ não possui valor fonético. $\mathrm{O}$ uso de $<$ gu $>$ e $<\mathrm{qu}>$ nos contextos apresentados é meramente um grafismo, representando apenas uma tradição gráfica latina. A alternância $<c>/<q u>$ diante de <a $>$ é, de acordo com Huber (1986, p. 51), freqüente em português desde o século XIII. Mattos e Silva $(1989$, p. 96) questiona se esses segmentos representariam a mesma

\begin{tabular}{|c|c|}
\hline $\begin{array}{c}\text { Manusinito 199 } \\
\text { doge (f. } 58 \mathrm{r}: 1.13)\end{array}$ & 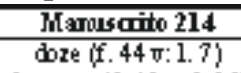 \\
\hline desprega (f. 63 r: 1.13$)$ & $48 \pi: 116$ \\
\hline
\end{tabular}

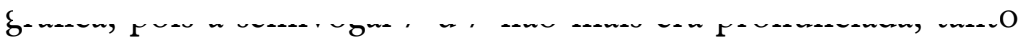

quando seguida por $\langle\mathrm{e}\rangle$ ou $\langle\mathrm{i}\rangle$, como também por $\langle\mathrm{a}\rangle$ ou $<\mathrm{o}>$.

Variações entre os grafemas $<$ ç $>$ e $<$ z $>$

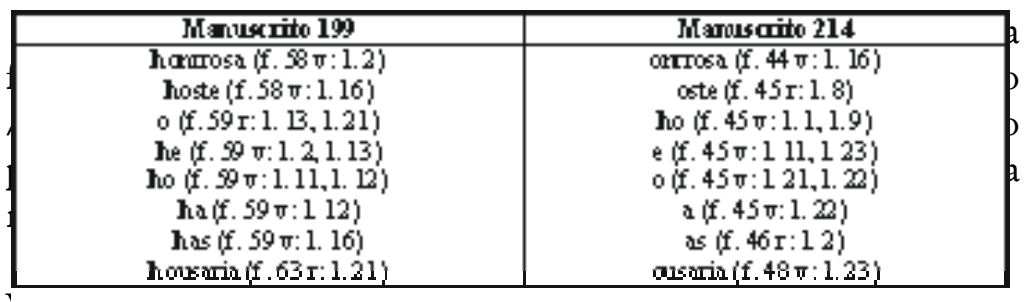

Em latim o grafema $<\mathrm{h}>$ representava uma aspiração. No processo evolutivo essa aspiração foi-se perdendo, o que gerou 
confusão no que se refere à grafia, pois ora se grafava com $\langle\mathrm{h}\rangle$ ora não. O que pose ser comprovado de acordo com os exemplos

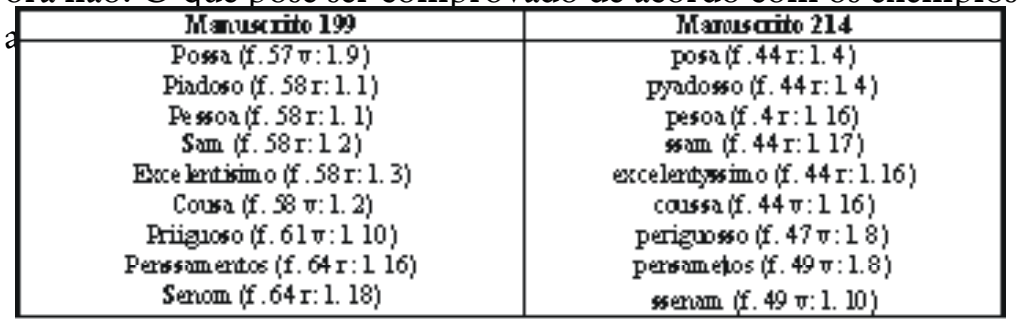

Variações entre os grafemas $<$ ss $>$ e $<$ s $>$

$\mathrm{O}$ fonema / s / deveria ser representado, etimologicamente, pelo grafema $<s>$ em posição inicial absoluta, em posição inicial de sílaba depois de vogal nasal e de consoante. Em posicão medial

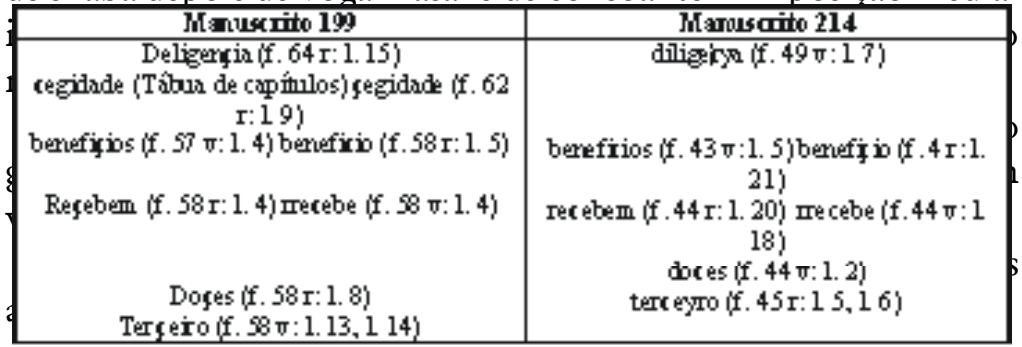

Variações entre os grafemas $<$ ç $>$ e $<c>$ 
Segundo Maia (1986, p. 442), o grafema <ç > desde o final do

\begin{tabular}{|c|c|}
\hline Manuscrito 199 & M: \\
\hline Reç ebem (f. $58 \mathrm{r}: 14)$ & recebem (f. $44 \mathrm{r}: 120$ ) \\
\hline desterro (f. $.58 \mathrm{r}: 1.6)$ & destero (f. $44 \mathrm{r}: 1.22$ ) \\
\hline Iresesti (f. $59 \mathrm{\pi}: 1.2$ ) & Regesti if. $45 \pi: 1.11$ ) \\
\hline Irefercm if. $59 \pi: 1$ i ) & Refey an if. $46 \mathrm{r}: 14$ ) \\
\hline mpuryer (f. $59 \pi: 1.20)^{\circ}$ & Reuryer $(f .46 r: 1.6)$ \\
\hline Morrer (f. $62 \mathrm{r}: 15$ ) & marer (f. $470: 121)$ \\
\hline Byco if $.62 \mathrm{r}: 1.21$ ) & nyco (f. $48 \mathrm{r}: 19$ ) \\
\hline hormondo (f. $62 \pi: 1.2$ ) & orrado if. $48 \mathrm{r}: 1$ lo) \\
\hline Hrame (f . $64 \mathrm{r}: 1.18$ ) & $\mathbf{r e m}(f .49 \pi: 1.10)$ \\
\hline
\end{tabular}

varıaçoes entre os gratemas <rr $>$ / $<\mathrm{r}>/<\mathrm{K}>$

Os grafemas $<\mathrm{r}>$ e $<\mathrm{rr}>$ representavam os fonemas vibrantes / $\mathrm{R} / \mathrm{e} / \mathrm{r} /$, respectivamente. A alternância entre os grafemas $<\mathrm{rr}><\mathrm{R}>$ e $<\mathrm{r}>$ parece ser meramente gráfica, dependendo da opção do copista. Geralmente o $<\mathrm{R}>$ tem valor de / r / dobrado : / rr /.

\section{CONSIDERAÇÕES FINAIS}

O estudo da variação grafemática em três tratados medievais portugueses, referentes a dois códices de datação variada: o manuscrito 199 é da segunda metade do século XV e o manuscrito 214 é da primeira metade do século XVI, revela que algumas oscilações na grafia são decorrentes de fatores diversos, tais 
como: influência etimológica, simples grafismo, ultracorreção ou influência fonológica.

NOTA

${ }^{1}$ Cota na Biblioteca Nacional de Lisboa: ALC 199 e ALC 214.

\section{REFERÊNCIAS}

CARDEIRA, Esperança Maria da Cruz Marreiros. Contributo para o estudo da norma ortográfica no scriptorium de Alcobaça (1431-1446). Lisboa: Faculdade de Letras, 1990.

GÃNDAVO, Pero de Magalhães de. Regras que ensinam a maneira de escrever e a ortografia da língua portuguesa: com o diálogo que adiante se segue em defensão da mesma língua. Lisboa: Biblioteca Nacional, 1981.

GUERRA, António Joaquim Ribeiro. Os escribas dos documentos particulares do Mosteiro de Santa Maria de Alcobaça - 1155-1200: Exercícios de análises de grafias. 1988. Dissertação (Mestrado em Paleografia e Diplomática) Faculdade de Letras, Universidade de Lisboa, Lisboa.

HAUY, Amini Boainain. História da língua portuguesa: séculos XII, XIII e XIV. São Paulo: Ática, 1989.

HUBER, Joseph. Gramática do português antigo. Tradução Maria Manuela Gouveia Delille. Lisboa: Calouste Gulbenkian, 1986. 
LEÃO, Duarte Nunes do. Ortografia e origem da língua portuguesa. Lisboa: Imprensa Nacional / Casa da Moeda, 1983. Introdução, notas e leitura por Maria Leonor Carvalhão Buescu.

MAIA, Clarinda de Azevedo. História do galego-português: estado lingüístico da Galiza e do Noroeste de Portugal desde o século XIII ao século XVI (com referência à situação do galego moderno).

MATTOS E SILVA, Rosa Virgínia. Estruturas trecentistas: elementos para uma gramática do português arcaico. Lisboa: Imprensa Nacional-Casa da Moeda, 1989.

. O português arcaico: fonologia. São Paulo/Salvador: Contexto/EDUFBa, 1991.

MICHAËLIS DE VASCONCELOS, Carolina. Lições de filologia portuguesa. Lisboa: Revista de Portugal, 1946.

OLIVEIRA, Fernão de. A gramática da linguagem portuguesa. Lisboa: Imprensa Nacional / Casa da Moeda, 1975. Introdução, leitura actualizada e notas por Maria Leonor Carvalhão Buescu.

PAIVA, Dulce de Faria. História da língua portuguesa: século XV e meados do século XVI. São Paulo: Ática, 1988.

QUEIROZ, Rita de Cássia Ribeiro de. "Dos benefícios de Deus", "Livro da consciência e do conhecimento próprio", "Da amizade e das qualidades do amigo": Edição e vocabulário onomasiológico de três tratados da obra ascético-mística "Castelo Perigoso" (Cód(s). ALC 199 e ALC 214. 2002. 475 f. Tese (Doutorado em Filologia e Língua Portuguesa) Faculdade de Filosofia, Letras e Ciências Humanas, Universidade de São Paulo, São Paulo.

SANTANA NETO, João Antônio de ; QUEIROZ, Rita de Cássia 
Ribeiro de. Variação grafemática no texto do "Castelo Perigoso". Estudos Lingüísticos, São Paulo, v. 28, p. 188-193, 1999.

SILVA NETO, Serafim da. História da língua portuguesa. Rio 
225 